\title{
Petroleum rock mechanics: An area worthy of focus in geo-energy research
}

\author{
Chunhe Yang, Jianjun $\operatorname{Liu}^{\circledR *}$ \\ Institute of Rock and Soil Mechanics, Chinese Academy of Sciences, Wuhan 430071, P. R. China
}

Cited as:

Yang, C., Liu, J. Petroleum rock mechanics: An area worthy of focus in geo-energy research. Advances in Geo-Energy Research, 2021, 5(4): 351-352, doi: 10.46690/ager.2021.04.01

Rock mechanics is a discipline that studies the stress, strain, failure, stability, and reinforcement of rocks under the action of external factors (such as load, fluid flow, and temperature changes). It is not only a branch of mechanics but also an interdisciplinary engineering subject. This discipline requires knowledge of applied mathematics, solid mechanics, fluid mechanics, geology, soil mechanics, and civil engineering, etc., and the main purpose is to solve engineering problems that arise from anthropogenic or natural processes concerning rock physics. Petroleum, a complex mixture of hydrocarbons that occurs in subsurface rocks in liquid, gaseous, or solid form, includes oil, natural gas, and the viscoelastic solid bitumen. Literally, "petroleum" means "rock oil" from Latin, in which the word "petra" means "rock" or "stone" while "oleum" corresponds to "oil".

The upstream of the petroleum industry mainly includes the processes of petroleum geophysical prospecting, drilling \& completion, fracturing, injection \& production, and storage. Each of the abovementioned phases is directly related to rock mechanics, and for some of these stages, the success of the project directly depends on the mechanical behavior of the subsurface rocks.

- In the stage of petroleum geophysical exploration, it is necessary to ascertain the intrinsic correlation between petrophysical properties and field parameters related to geophysical exploration methods, e.g., how rock elastic moduli will affect wave propagation during acoustic porosity logging, which is conducive to improving the accuracy of reservoir identification and explaining the oil and gas occurrence states as well as physical parameters of the reservoir.

- In the drilling and completion stage, rock mechanics is the main theoretical basis to ensure wellbore stability and prevent sand production or casing damage. At this stage, it is necessary to establish mechanical models through analytical or numerical methods to reveal the mechanical mechanisms of wellbore instability, sand production, and casing damage for various well types such as vertical, inclined, horizontal, and lateral wells. Consequently, controlled measurements can be made to ensure the stability and integrity of the borehole.

- Fracturing is a key technology for the efficient development of low-permeability oil and gas reservoirs. Based on the theories of rock fracture mechanics and fluid mechanics, the evaluation metric of rock fracability can be proposed, the crack propagation pattern of complex reservoir rocks during hydraulic fracturing can be revealed, and the process and parameters of hydraulic fracturing can be optimized based on computer simulation of hydraulic fracturing.

- Fluid injection and production can usually be regarded as a pure fluid transport problem in reservoir engineering contexts. But the reservoir rock is, after all, an elastoplastic material rather than a rigid body. Therefore, in order to accurately predict oil and gas production and optimize injection and production protocols, reservoir thermo-hydromechanical coupling theory has been incorporated into reservoir simulation and geo-stress evolution analysis. By formulating different rock constitutive models, fluid transport models, and multi-physics coupling algorithms,

\section{Yandy
Scientific}

Press

${ }^{*}$ Corresponding author.

E-mail address: chyang@whrsm.ac.cn (C. Yang); jjliu@whrsm.ac.cn (J. Liu).

2207-9963 @ The Author(s) 2021.

Received August 20, 2021; revised August 23, 2021; accepted August 23, 2021; available online August 25, 2021.
} 
integrated simulations of reservoir fluid pressure and solid stress fields can be achieved.

- Large-scale underground gas storage is an important measure to ensure the safe production of oil and gas. In the stage of site selection and operating parameter design, it is necessary to study the sealing efficiency of the caprock and the stability of the nearby faults based on rock mechanics and fluid-solid coupling theories, so as to determine the safety window for all the operating parameters during gas storage.

- In the design of salt cavern gas storage projects, rock mechanics analysis is necessary for salt cavern stability evaluation, injection pressure optimization, and geological disasters prevention, such as land subsidence and induced seismicity. In addition to the issues mentioned above, natural gas hydrate extraction, enhanced heavy oil recovery, and shale gas/oil reservoir development also involve complex thermal-hydraulic-mechanical-chemical coupling mechanisms.

Nowadays, the development of oil and gas reservoirs is getting deeper and deeper. For example, some wells in the Tarim Oilfield in Xinjiang, have been drilled more than 8,000 meters in depth. At the same time, rock mechanics is playing an increasingly important role in the oil and gas industry with the increasing development of unconventional oil and gas reservoirs. In the coming period, the research of petroleum rock mechanics will focus on:

- Rock mechanical properties and behaviors under high temperature and high pressure and their influences on reservoir productivity;

- The propagation and control of hydraulic fractures in complex reservoirs, as well as new and efficient fracturing methods for shale oil and gas reservoirs;
- Improved reservoir rock thermal-hydraulic-mechanicalchemical multi-physics coupling algorithms;

- High temperature and high pressure physical experiments of reservoir rock under multi-physics conditions;

- Accurate and efficient computational algorithms and simulator development for multi-physics coupling problems.

Certainly, with the in-depth intersection and integration of various related disciplines, 3D printing technology, artificial intelligence algorithm and other technologies have been introduced into petroleum rock mechanics, and numerous new research directions are emerging.

The papers published in this virtual special issue are closely related to petroleum rock mechanics and have important significance in both theoretical and practical aspects. In these 16 articles, the research on the modeling and simulation of permeability variation is mainline. Coupled multi-scale and multi-physics characterization, as well as the related simulation methods are the focus of attention. The purpose of the included researches is to discover and explain the mechanisms governing the exploitation of geological resources and ultimately provide guidance for engineering practice.

We hope that while reading and citing these papers, colleagues will think profoundly and deepen the research in this area, and jointly promote the development of petroleum rock mechanics.

\section{Conflict of interest}

The authors declare no competing interest.

Open Access This article is distributed under the terms and conditions of the Creative Commons Attribution (CC BY-NC-ND) license, which permits unrestricted use, distribution, and reproduction in any medium, provided the original work is properly cited. 